\title{
Vehículos híbridos, una solución interina para bajar los niveles de contaminación del medio ambiente causados por las emisiones provenientes de los motores de combustión interna
}

\section{Hybrid vehicles, an interim solution to lower levels of environmental pollution caused by emissions from internal combustion engines}

\author{
Darwin Gregorio Chele Sancan \\ Universidad Internacional del Ecuador, Ecuador
}

Autor para correspondencia: darwinchelesancan@ @otmail.com

Fecha de recepción: 15 de octubre de 2017 -Fecha de aceptación: 30 de noviembre de 2017

Resumen: La calidad del aire en el mundo se ve afectada por las emisiones del parque automotor donde el principal combustible para su funcionamiento sigue siendo los derivados del petróleo, la gasolina y el diésel, según datos del Instituto Nacional de Estadísticas y Censos (INEC) hasta el año 2015 fueron matriculados 1925368 vehículos en el Ecuador y cuyas emisiones de gases está deteriorando la calidad de vida de sus habitantes. Entre los daños causados por los gases provenientes de los residuos de la combustión destacan el calentamiento global, daño a la capa de ozono y daño a la salud de los seres vivos. En los últimos cien años los vehículos autopropulsados por medio de un motor de combustión interna (MCI) han dominado la industria de la transportación, hoy en día los esfuerzos por reducir los niveles de contaminación han dado paso a que los fabricantes de vehículos investiguen sobre una nueva alternativa de transportación y de combustibles alternativos, que sean eficientes y reduzcan la emisión de gases contaminantes. Los combustibles alternativos no contaminan el medio ambiente cuando se los fabrica ni cuando se los utiliza, como lo hacen los combustibles fósiles, otro combustible es a base del hidrógeno los cuales utilizan celdas electroquímicas como fuente de energía y que no tienen emisiones de carbono. La electricidad también paso a ser una fuente de energía para los vehículos la cual proviene de baterías que convierten la energía química en electricidad. Actualmente se ha dado paso a los vehículos eléctricos en especial a los denominados vehículos eléctricos híbridos HEV por sus siglas en inglés (Hybrid Electric Vehicle) cuya principal fuente de energía para su desplazamiento es un motor eléctrico el cual se complementa a un MCI, los vehículos híbridos están identificados como una solución interina, por lo pronto la más viable, fundamentalmente para resolver los problemas asociados con los vehículos propulsados por un MCI. El MCI de un vehículo híbrido produce menos emisiones de gases contaminantes comparado con un vehículo convencional de similares características debido a que el motor es un poco más pequeño y no es utilizado directamente para propulsión del vehículo.

Palabras claves: contaminación; transportación; combustibles; nuevas alternativas; híbrido

Abstract: The world air quality is affected by emissions from the vehicles where the main fuel for its operation continues being derived from oil, gasoline and diesel, according to the Instituto Nacional de Estadísticas y Censos (INEC) until 2015, 1925368 vehicles were registered in Ecuador and the emissions are deteriorating the quality of populations life. Among the damages caused by the gases from the combustion residues are the global warming, damage to the ozone layer and damage to the health of living beings. 
In the last hundred years, self-propelled vehicles propelled by internal combustion engine (ICE) have dominated the transportation industry, today efforts to reduce pollution levels have led vehicle manufacturers to research a new transportation alternative and alternative fuels that are efficient and reduce the emission of polluting gases. Alternative fuels do not pollute the environment when they are made, or used, as fossil fuels do, other hydrogen-based fuels that use electrochemical cells as a source of energy and have no carbon emissions. Electricity also happens to be a source of energy for vehicles which comes from batteries that convert chemical energy into electricity. Currently, electric vehicles have been given special attention to the called Hybrid electric vehicles HEV which main source of energy for its displacement is an electric motor which complements an ICE, the hybrid vehicles are identified as a temporary solution, for the moment is being the most viable, fundamentally to solve the problems associated with vehicles powered by an ICE. The ICE of a hybrid vehicle produces low emissions of polluting gases compared to a conventional vehicle of similar characteristics because the engine is smaller and is not used directly for vehicle propulsion.

Key words: fuel; pollution; hybrid; new alternatives; transportation

\section{Introducción}

\section{Problema}

Los sistemas convencionales de producción de energía utilizado por décadas en los vehículos autopropulsados, provienen de los combustibles fósiles, sin embargo en los últimos años se ha presentado una creciente preocupación por el ahorro de combustibles y el cuidado del medio ambiente, lo que ha dado paso a una nueva fuente de energía para la propulsión de los vehículos, el cual consiste en un motor eléctrico en combinación con un motor de combustión interna, a este nuevo sistema se lo conoce con el nombre de Híbrido, cuyo principal objetivo es reducir las emisiones y el consumo de combustibles fósiles.

El aire es esencial para las formas de vida existentes en la Tierra al igual que la capa atmosférica que los protege, su alteración modifica la calidad del aire y puede causar cambios climáticos, los gases expulsados por los MCI provenientes de la combustión de la gasolina o el diésel contribuyen a la generación del efecto invernadero, dañando la capa de ozono y causando el calentamiento global, además el petróleo es un recurso natural que en un futuro no muy lejano no se tendrá al alcance.

Hay una variedad de contaminantes atmosféricos donde las principales fuentes son el área industrial y el área automotriz, entre estos contaminantes destacan según Querol, Viana, Moreno, \& Alastuey (2012);

El dióxido de carbono (CO2), el monóxido de carbono (CO), el óxido de azufre (SO2), los óxidos de nitrógeno (NOx, NO, NO2) .... El dióxido de carbono (CO2) ... no se dejan sentir a nivel local o regional sino en su contribución planetaria al efecto invernadero y al calentamiento global de la atmósfera (pág. 20).

A esto se adiciona el material particulado (PM) los cuales se encuentran en los residuos de la combustión de los motores a diésel (Sánchez, 2009). Todo esto contribuye a tener elevados niveles de contaminación en el aire que respiramos provocando problemas cardiorrespiratorios. 
Según un estudio realizado por Chan (2002) citado en Caiying, Peng, \& Tao (2011) sobre el incremento de la población y de los vehículos, en los próximos cincuenta años la población global se podría incrementarse de seis billones en el año 2000 a diez billones en el año 2050, generando un incremento del parque automotor de setecientos millones a dos billones y medio de unidades. Si todas estas unidades funcionaran con un motor de combustión interna a gasolina o diésel estos combustibles se agotarían rápidamente, con graves consecuencias por la emisión de gases contaminantes, por lo que la conservación de la energía y la protección del medio ambiente es un tema de creciente interés a nivel mundial.

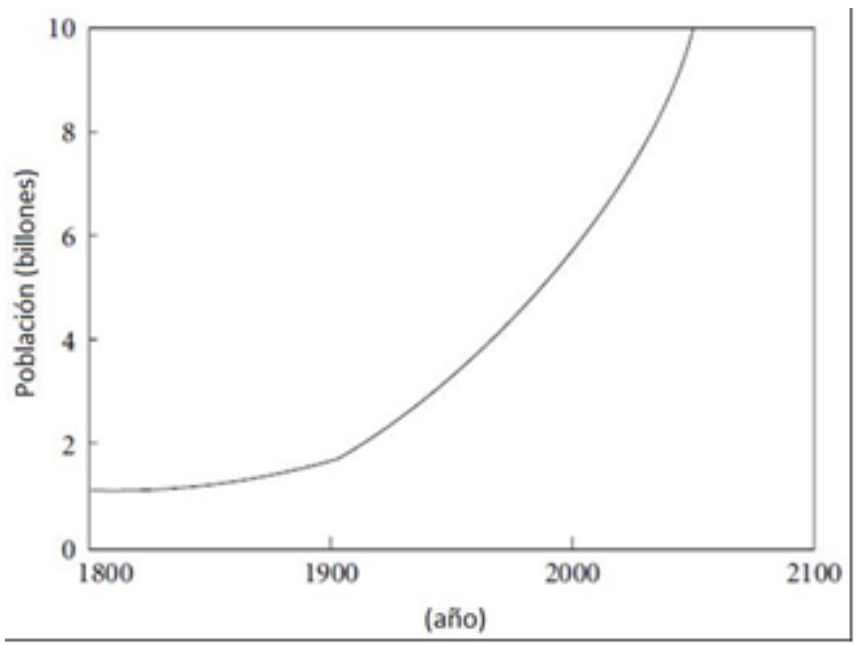

Figura 1. Crecimiento de la población.

Fuente: A Comprehensive Overview of Hybrid Electric Vehicles; Caiying, Peng, \& Tao (2011).

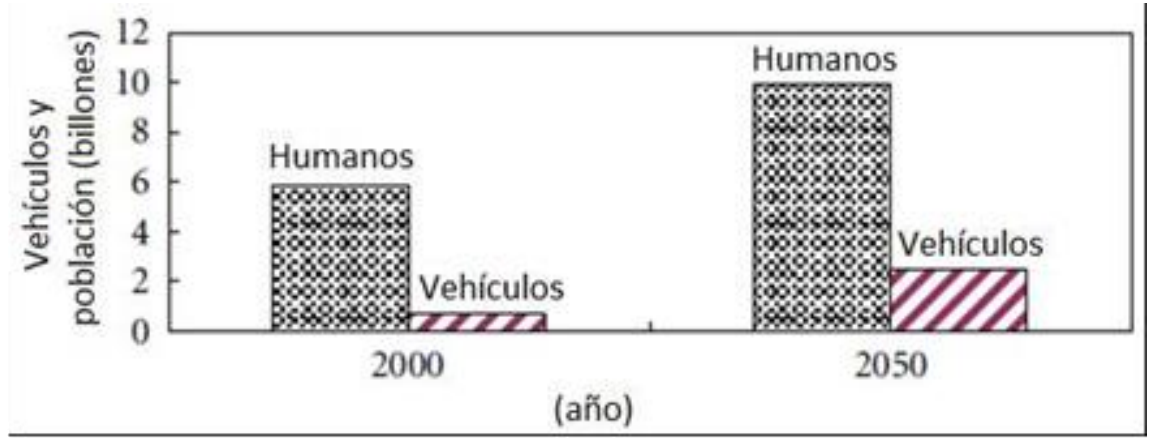

Figura 2. Vehículos y población.

Fuente: A Comprehensive Overview of Hybrid Electric Vehicles; Caiying, Peng, \& Tao (2011).

\section{Reducción de gases contaminantes}

La reducción de los gases contaminantes como el CO2, CO, NOx, NO2 y PM pueden tener dos alternativas de solución: la tecnológica y la no tecnológica. En el caso de la intervención tecnológica uso del biodiesel puede reducir las emisiones de $\mathrm{CO} 2$ pero puede aumentar las emisiones del NOx, utilizar filtros de partículas para reducir las emisiones de PM conlleva a un incremento de NO2 primario e incentivar la compra de vehículos con bajas emisiones de $\mathrm{CO} 2$ puede conducir a la compra de vehículos a diésel lo cual puede incrementar las emisiones de PM y NO2. 
En si la intervención tecnológica encamina a los fabricantes de vehículos a adaptarse a las nuevas normas y a los planes de mejora de la calidad del aire. La intervención no tecnológica se basa en la reducción de la intensidad y la densidad del tráfico. Como medida complementaria pueden ser las estrategias a medio y largo plazo con miras a tener un parque automotor más ecológico en lo que se refiere a vehículos privados y públicos, esta última con actuación inmediata (Castells, 2012; Otterbach, 2014; Querol, Viana, Moreno, \& Alastuey, 2012).

Un auto eléctrico aún no es la solución para a los problemas de contaminación, debido a que se necesita, para la generación de la electricidad plantas termoeléctricas las cuales utilizan combustibles fósiles, además de que presentan inconvenientes con su autonomía eléctrica limitada y la falta de estaciones públicas de recarga, misma que se la realiza en tiempos relativamente largos. Una solución a estos inconvenientes fue el desarrollo de los vehículos híbridos (Otterbach, 2014).

\section{Gases contaminantes}

En los MCI a gasolina se produce una combustión del combustible, el cual es una mezcla de hidrocarburos compuesto principalmente por carbono e hidrógeno y el comburente que es el oxígeno contenido en el aire. En el caso de una combustión perfecta, mezcla estequiométrica, 1 gramo de combustible por 14,7 gramos de aire, el carbono se une al oxígeno del aire para formar dióxido de carbono (CO2) y el hidrógeno se une con el oxígeno formando agua $(\mathrm{H} 2 \mathrm{O})$, además de obtenerse el nitrógeno (N2), al no obtenerse la proporción de aire - combustible ideal surgen otras sustancias contaminantes como:

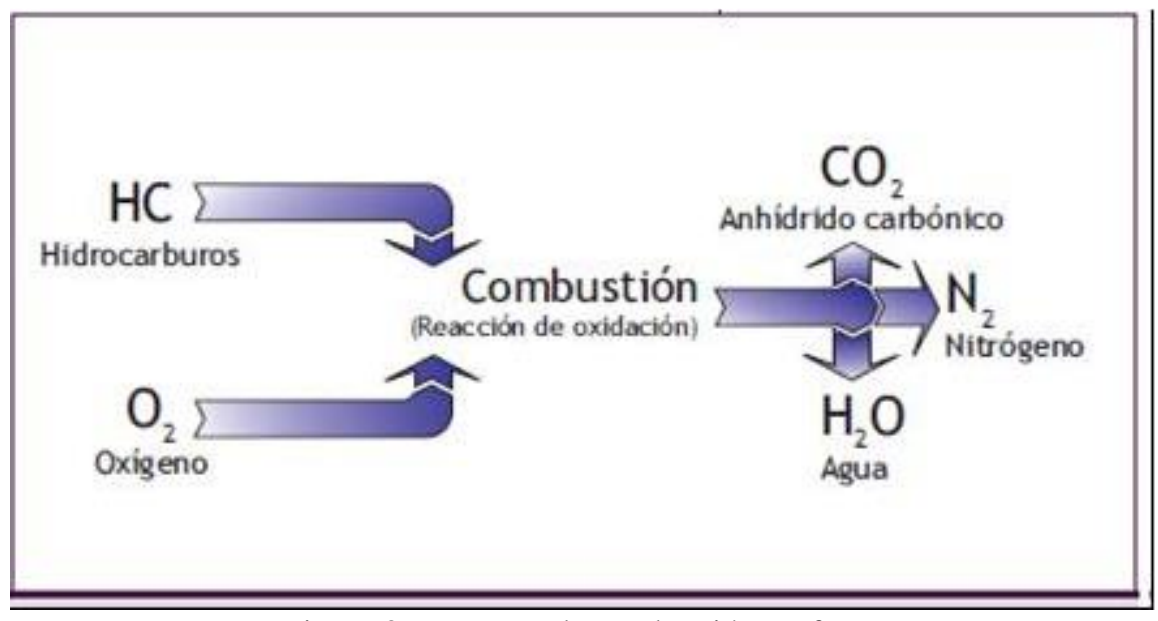

Figura 3. Esquema de combustión perfecta.

Fuente: Sistemas Auxiliares del Motor, Sánchez (2009).

- El monóxido de carbono (CO), compuesto conocido, tóxico, incoloro e inodoro que en contacto con el aire libre se une con el oxígeno para formar el dióxido de carbono (CO2). Es altamente tóxico y puede llegar a ser mortal, bloquea el transporte de oxígeno por parte de los glóbulos rojos. Se produce por una combustión incompleta de combustible con carbono (Sánchez, 2009).

Tabla 1. Efectos del monóxido de carbono 


\begin{tabular}{cc}
\hline Nivel (ppm) & Efecto \\
\hline $\mathbf{2 0 0}$ por 3 horas ó 600 por una hora & Dolor de cabeza \\
500 por una hora ó 1000 por 30 minutos & Mareos, zumbido de oídos, náuseas \\
$\mathbf{1 5 0 0}$ por una hora & Sumamente peligroso \\
4000 & Colapso, inconsciencia, muerte \\
\hline
\end{tabular}

Fuente: Determinación de las ventajas ambientales que presenta un vehículo híbrido respecto a un vehículo normal de similares características, Cajamarca Tigre \& García Toledo (2010).

El dióxido de carbono (CO2) es un gas incoloro, inodoro y en principio no tóxico, un incremento desmesurado puede producir variaciones climáticas a gran escala.

- Hidrocarburos (HC) no quemados, son causados por un exceso de combustible en la mezcla o por una combustión incompleta y la vaporización del combustible. Su olor es penetrante y su color azulado.

- Óxido nítrico (NOx) se forma a partir del nitrógeno contenido en el aire procedente de la combustión. La gran temperatura generada por la combustión provoca, en condiciones de exceso de oxígeno, la formación de óxidos de nitrógeno que además de ser contaminante disminuyen el oxígeno necesario para completar la combustión.

- Partículas de hollín (PM), es generada en su mayor parte por los motores a diésel, su condición de partículas sólidas la convierte en sucia y molesta pudiendo ocasionar taponamiento de las vías respiratorias.

- Dióxido de azufre (SO2), es un gas incoloro de olor penetrante y no combustible que interviene en una medida muy reducida en los gases de escape, propicia las enfermedades de las vías respiratorias, se puede bajar los niveles de emisión del dióxido de azufre reduciendo el contenido de azufre en el combustible.

\section{Vehículos híbridos}

Los vehículos eléctricos EV (Electric Vehicles) por sus siglas en inglés, están clasificados en tres tipos: vehículos puramente eléctricos (PEVs), vehículos eléctricos híbridos (HEVs) y los vehículos de eléctricos de celdas de combustible (FCEVs), sus principales características se muestran en la siguiente tabla.

Tabla 2. Principales características de los tres tipos de vehículos eléctricos.

\begin{tabular}{cccc}
\hline Tipos de EV & PEV & HEV & FCEV \\
\hline Fuente de energía & Batería & $\begin{array}{c}\text { Batería/ultra capacitor } \\
\text { Motor de combustión } \\
\text { interna }\end{array}$ & Celdas de combustible \\
Técnica de propulsión & Motor eléctrico & Motor eléctrico & Motor eléctrico \\
& & Motor de combustión & interna \\
\hline
\end{tabular}




\begin{tabular}{|c|c|c|c|}
\hline Características & $\begin{array}{c}\text { Cero emisiones } \\
\text { Rango de conducción } \\
\text { corto } \\
\text { Costos iniciales altos }\end{array}$ & $\begin{array}{c}\text { Bajas emisiones } \\
\text { Rango de conducción } \\
\text { largo } \\
\text { Complejo }\end{array}$ & $\begin{array}{c}\text { Cero emisiones } \\
\text { Costos iniciales altos } \\
\text { Rango de conducción } \\
\text { medio }\end{array}$ \\
\hline Técnicas principales & $\begin{array}{l}\text { Control del motor } \\
\text { eléctrico } \\
\text { Gestión de batería } \\
\text { Estrategia de carga }\end{array}$ & $\begin{array}{l}\text { Control del motor } \\
\text { eléctrico } \\
\text { Gestión de batería } \\
\text { Gestionamiento } \\
\text { múltiple de fuentes de } \\
\text { energía y eficiencia } \\
\text { optima del sistema } \\
\text { Componentes de } \\
\text { grandes }\end{array}$ & $\begin{array}{l}\text { Central de } \\
\text { procesamiento de } \\
\text { combustible } \\
\text { Sistema de combustible } \\
\text { Costos de las celdas de } \\
\text { combustible }\end{array}$ \\
\hline Freno regenerativo & $\mathrm{Si}$ & $\mathrm{Si}$ & $\mathrm{Si}$ \\
\hline
\end{tabular}

Fuente: A Comprehensive Overview of Hybrid Electric Vehicles; Caiying, Peng, \& Tao (2011).

Muchas fábricas de automóviles empezaron a producir autos eléctricos los cuales estaban disponibles tanto para la venta o el alquiler al público en general, pero debido a la baja demanda, frecuentemente se suspende su producción, por el inconveniente que presentan con su autonomía, lo cual significó un verdadero reto en la investigación y el desarrollo de una tecnología que permita obtener vehículos con cero emisiones. La industria automotriz japonesa, específicamente la marca Toyota y Honda, introdujeron al mercado en el año 1990 los vehículos híbridos con los modelos Prius e Insight respectivamente. Honda utilizo la configuración paralela y Toyota la configuración serie - paralelo en sus vehículos híbridos, estos modelos representan una moderna escuela para este tipo de vehículos (Husain, 2011).

Los vehículos híbridos están compuestos por un motor de combustión interna a gasolina más pequeño y ligero, un motor eléctrico, generador, transmisión, batería para el motor eléctrico y un tanque de combustible pequeño para el MCI, el término más empleado para referirse a estos vehículos es HEV, en otras palabras, estos vehículos combinan dos métodos de propulsión, en uno su fuente de energía esta almacenada y el otro transforma la energía del combustible fósil. La electrónica en estos vehículos permite que el motor eléctrico actúe como motor y como generador, al disminuir la marcha del vehículo, por medio del freno, el motor eléctrico actúa como generador cargando las baterías, cuando se acelera el motor eléctrico absorbe la energía de las baterías, en carreteras el motor de combustión no necesita de toda su energía y se puede recargar las baterías mientras avanza. Los fabricantes de vehículos los consideran como el paso intermedio para pasar de vehículos con MCI a vehículos con motores eléctricos.

Los vehículos híbridos según se dispongan el motor de combustión y el eléctrico se clasifican en tres tipos: paralelo, serie y combinado. En serie solo el motor eléctrico tiene control sobre la tracción y el MCI recargas las baterías cuando sea necesario. Una configuración en serie presenta la ventaja de que reduce las emisiones contaminantes y el consumo y su transmisión es sencilla, pero en cambio tiene la desventaja de que sus baterías son de mayor tamaño, menor rendimiento en el transito energético mecánico-eléctrico-mecánico y mayor motor eléctrico; el paralelo tiene control de la tracción tanto el MCI y el motor eléctrico por separado o los dos a la vez (según la velocidad y potencia requerida). 

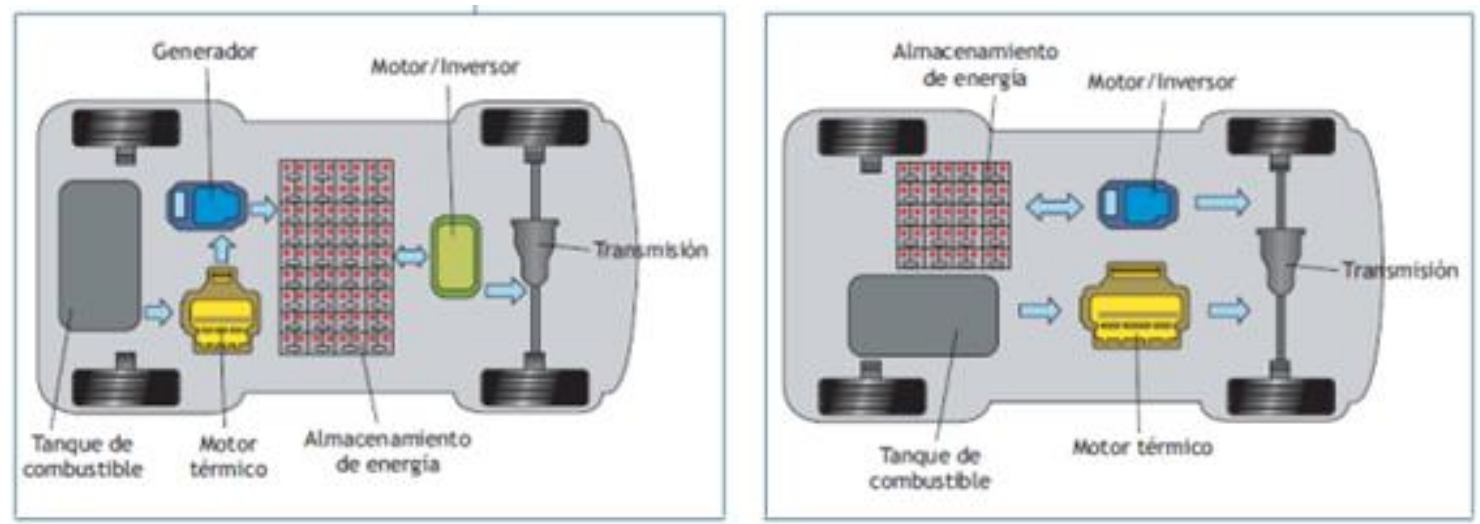

Figura 4. Configuración en serie y en paralelo de un HEV.

Fuente: Motores. Escudero, González, Rivas, \& Suárez (2009).

El combinado incorpora las características de la configuración en serie y del paralelo, presenta una conexión mecánica adicional entre el motor de combustión interna y la transmisión, comparada con la configuración en serie, también tiene un generador adicional entre el motor de combustión y el convertidor de energía, comparado con la configuración en paralelo, aun cuando la complejidad de sus componentes y los costos de los mismos es más flexible en su control.

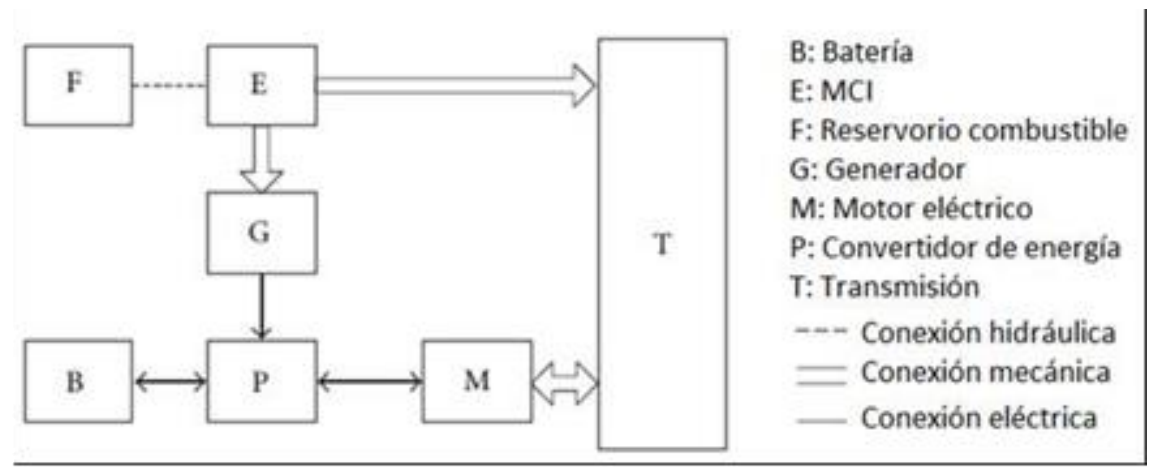

Figura 5. Configuración combinada de un HEV.

Fuente: A Comprehensive Overview of Hybrid Electric Vehicles; Caiying, Peng, \& Tao (2011).

La siguiente tabla enlista la configuración de algunos modelos de EV.

Tabla 3. Configuraciones típicas de HEV.

\begin{tabular}{llll}
\hline Modelo & Configuración & Fabricante & Año \\
\hline Prius & Combinado & Toyota & 1997 \\
Insight & Paralelo & Honda & 1999 \\
Tino & Combinado & Nissan & 2000 \\
Civic & Paralelo & Honda & 2001 \\
Lexus LS 600h & Combinado & Toyota & 2007 \\
Toyota Auris & Combinado & Toyota & 2010 \\
Lexus CT 200h & Combinado & Lexus & 2011 \\
\hline
\end{tabular}

Fuente: A Comprehensive Overview of Hybrid Electric Vehicles; Caiying, Peng, \& Tao (2011).

El MCI de un HEV no obedece a la definición habitual de un motor a gasolina como lo estableció August Otto, sino que utiliza un ciclo termodinámico que inicio el inglés Atkinson y 
lo puso a punto el americano Millar por el año 1940. Al ciclo Millar también se lo conoce como el "ciclo de cinco tiempos" lo que permite obtener un rendimiento superior al de los motores de ciclo Otto, aunque su potencia específica es débil esta es compensada por la potencia del motor eléctrico.

Tabla 4. Características comparadas de un vehículo híbrido y otro convencional de similares prestaciones (Extraídos catalogo Toyota)

\begin{tabular}{|c|c|c|}
\hline Características & Híbrido (Prius) & Convencional (Avensis) \\
\hline \multicolumn{3}{|c|}{ Motor de gasolina } \\
\hline Cilindrada $\left(\mathrm{cm}^{3}\right)$ & 1497 & 1794 \\
\hline Relación de compresión & $13,0: 1$ & $10,0: 1$ \\
\hline Potencia máxima (CV/rpm) & $78(57 \mathrm{KW}) / 5000$ & $129(95 \mathrm{KW}) / 6000$ \\
\hline Par máximo (N.m/rpm) & $115 / 4000$ & $170 / 4200$ \\
\hline Velocidad máxima $(\mathrm{km} / \mathrm{h})$ & 170 & 200 \\
\hline Aceleración 0-100km/h (s) & 10,9 & 10,3 \\
\hline Coeficiente aerodinámico & 0,26 & 0,28 \\
\hline \multicolumn{3}{|c|}{ Motor eléctrico } \\
\hline Tensión nominal (V) & 500 & - \\
\hline Potencia máxima (CV/rpm) & $68(50 \mathrm{KW}) / 1200-1540$ & - \\
\hline Par máximo (N.m/rpm) & $400 / 0-1200$ & - \\
\hline \multicolumn{3}{|c|}{ Batería híbrida Ni-metal hidruro } \\
\hline Tensión nominal (V) & 201,6 & - \\
\hline Capacidad (Ah) & $6,5(3 \mathrm{~h})$ & - \\
\hline Peso $(k g)$ & 39 & - \\
\hline \multicolumn{3}{|c|}{ Rendimiento HSD (híbrido) } \\
\hline Potencia máxima (CV/rpm) & $111(82 \mathrm{KW}) /$ desde $85 \mathrm{~km} / \mathrm{h}$ & \\
\hline Par máximo (N.m/rpm) & 478 / hasta $22 \mathrm{~km} / \mathrm{h}$ & \\
\hline \multicolumn{3}{|c|}{ Datos ambientales } \\
\hline $\begin{array}{l}\text { Consumo combinado }(1 / 100 \\
\mathrm{km} / \mathrm{h})\end{array}$ & 4,3 & 7,2 \\
\hline Consumo urbano $(1 / 100 \mathrm{~km} / \mathrm{h})$ & 5,0 & 9,4 \\
\hline $\begin{array}{l}\text { Emisión } \mathrm{CO}_{2} \text { combinado (g } \\
\left.\qquad \mathrm{CO}_{2} / \mathrm{km}\right)\end{array}$ & 104 & 171 \\
\hline $\begin{array}{l}\text { Emisión } \mathrm{CO}_{2} \text { urbano }\left(\mathrm{g} \mathrm{CO}_{2} /\right. \\
\mathrm{km})\end{array}$ & 115 & 223 \\
\hline $\mathrm{CO}(\mathrm{g} / \mathrm{km})$ & 0,18 & 0,48 \\
\hline Hidrocarburos $(\mathrm{g} / \mathrm{km})$ & 0,02 & 0,03 \\
\hline $\mathrm{NO}_{\mathrm{x}}(\mathrm{g} / \mathrm{km})$ & - & 0,05 \\
\hline Partículas $(\mathrm{g} / \mathrm{km})$ & - & - \\
\hline Ruido dB (A) & 19 & 73 \\
\hline
\end{tabular}

Fuente: Energía y transporte. Castells (2012) 


\section{Conclusión}

El uso de combustibles alternativos que ayuden a solventar la crisis energética y el cuidado del medio ambiente es un tema de mucha importancia a nivel mundial, motivo por el cual se ha desarrollado algunas alternativas y fuentes de energía para la transportación, muchas de las cuales aún siguen en desarrollo debido a sus altos costos y cortos recorridos que se pueden obtener con dichas fuentes.

Los vehículos híbridos son hasta el momento una alternativa para satisfacer las necesidades de los consumidores a la vez que se obtiene una mejor economía de combustible y bajas emisiones de gases contaminantes, de los cuales se trató en el presente artículo, además de presentar ciertas características de los EVs y las configuraciones de los HEVs, aunque se continua utilizando motores de combustión interna su principal fuente para la propulsión del vehículo es un motor eléctrico, sin que esto signifique tener baterías de grandes dimensiones, debido a esto sus fabricantes lo consideran el paso intermedio entre los vehículos con MCI y los vehículos puramente eléctricos.

\section{Bibliografía}

Aishwarya, P., \& Hari Om, B. (2014). A Review of Optimal Energy Management Strategies for Hybrid Electric Vehicle. International Journal of Vehicular Technology, 1-19. Obtenido de https://www.hindawi.com/journals/ijvt/2014/160510/abs/

Caiying, S., Peng, S., \& Tao, G. (2011). A Comprehensive Overview of Hybrid Electric Vehicles. International Journal of Vehicular Technology, 1-7. Obtenido de https://www.hindawi.com/journals/ijvt/2011/571683/abs/

Cajamarca Tigre, D. A., \& García Toledo, V. E. (Julio de 2010). Determinación de las ventajas ambientales que presenta un vehículo híbrido respecto a un vehículo normal de similares características. Obtenido de Universidad Politécnica Salesiana: http://dspace.ups.edu.ec/handle/123456789/4843

Castells, X. E. (2012). Energía y transporte. Madrid: Ediciones Díaz de Santos. Obtenido de http://www.ebrary.com

Escudero, S., González, J., Rivas, J. L., \& Suárez, A. (2009). Motores. Madrid: Macmillan Iberia. Obtenido de http://www.ebrary.com

Fuhs, A. E. (2009). Hybrid Vehicles and the future of personal transportation. Unite States of America: Taylor \& Francis Group. Obtenido de https://goo.gl/2VLnjS

Husain, I. (2011). Electric and Hybrid Vehicles (Second Edition ed.). United States of America: Taylor \& Francis Group. Obtenido de https://goo.gl/rkDF22

Jiménez Padilla, B. (2012). Técnicas básicas de electricidad de vehículos (MF0624_1). Málaga: IC Editorial. Obtenido de http://www.ebrary.com 
Khajepour, A., Fallah, S., \& Goodarzi, A. (2014). Electric and hybrid. Technologies, modeling and control: a mechatronic approach. United Kingdom: John Wiley \& Sons Ltd. Obtenido de http://www.ebrary.com

Mashadi, B., \& Crolla, D. (2012). Vehicle powertrain systems. United Kingdom: John Wiley \& Sons, Ltd. Obtenido de http://www.ebrary.com

Muhammad, A. (2017). Advanced Charging System for Plug-in Hybrid Electric Vehicles and Battery Electric Vehicles. InTech, 63-81. Obtenido de https://www.intechopen.com/books/howtoreference/hybrid-electric-vehicles/advancedcharging-system-for-plug-in-hybrid-electric-vehicles-and-battery-electric-vehicles

Orie, C. J., \& Nwatu, Q. I. (2011). Challenges of Energy Saving Crisis as a Panacea to Hybrid. Mediterranean Journal of Social Sciences, 2(6), 67-75. Obtenido de https://goo.gl/yDgbH7

Otterbach, D. H. (2014). Energía y calentamiento global. ¿Cómo asegurar la supervivencia de la humanidad? México: Grupo Editorial Patria. Obtenido de http://www.ebrery.com

Pavan, R. (2014). A review on hybrid vehicles. Impact Journals, 2(5), 59-64. Obtenido de www.impactjournals.us

Querol, X., Viana, M., Moreno, T., \& Alastuey, A. (2012). Bases científico-técnicas para un Plan Nacional de Mejora de la Calidad del Aire. Madrid: Cyan, Proyectos Editoriales, S.A. Obtenido de http://www.ebrary.com

Raines, G. B. (2009). Electric vehicles : technology, research, and development. New York: Nova Science Publishers, Inc. Obtenido de http://www.ebrary.com

Sánchez, E. (2009). Sistemas auxiliares del motor. Madrid: Macmillan Iberia, S.A. Obtenido de http://www.ebrary.com

Smith, T. L. (15 de Enero de 2017). The Hybrid Vehicle and Alternative Fuel Report. Obtenido de Washington State Department of Transportation: https://www.wsdot.wa.gov/sites/default/files/2017/01/13/HybridReportJan1520157.pdf 